\title{
Quantitative Descriptions of Drainage Processes on Irregular Surface of Road Segments Based on Advanced Computer Simulation Techniques (II)
}

\author{
-Simulation Model for Description of Drainage Process on Irregular Road Surface-
}

\author{
Masami Shiba ${ }^{1}$
}

\begin{abstract}
This paper presents a simple system of quantitative descriptions of drainage processes on irregular surfaces of road segments based on computer simulation techniques, and it proposes an alternative flow model which more closely takes into account linked geomorphic and hydrologic properties. In earlier papers, the author formulated such a model in the one-dimensional manner. The approach employed here is extension of this model into two dimensions. The model derived here represents the mode of accumulation of water in depressions on the irregular surface and the development of oversurface flow. The surface flow system is simulated by a simple storage model. The assumptions behind a model postulated as describing the model of accumulation of water on irregular surfaces are that the infiltration capacity of soil is exceeded and the rainfall continues. Accordingly, the residual water ration (the depression-storage of irregular surfaces) can be derived in a simple way. In order to verify the applicability of these models for practical purpose, several types of road surface consolidated by different combinations of operations and machinery were analyzed.
\end{abstract}

Keywords: Forest road; Irregular surface; Drainage process; Surface flow; Computer simulation; Residual water ratio; Consolidation

\section{Introduction}

Perhaps the most ubiquitous process operating on a soil road surface is erosion and the transport of running water on the road. In an optimal design of surface drainage systems, it becomes a matter of importance how to provide a framework for understanding the behavior of rainfall and runoff and their relation to surface conditions (BC Code, 1995; Dennis et al., 1996; Silversides 1989).

Rain falling on an irregular surface disposes itself in a number of different ways. If the soil is not saturated, moisture will infiltrate into the ground at a rate controlled by the soil texture, material cover, and degree of saturation. Initially the rate of infiltration is rapid, but after a period of time the rate approaches an asymptotic limit, defined as the 'infiltration capacity' of a particular soil (Coker et al., 1993; Visser et al., 1993).

The infiltration capacity is the maximum sustained rate at which a particular soil will absorb water. If infiltration capacity of the soil is exceeded and rain continues, water will collect in depressions on the surface of the ground. These depressions may be minute or large in size, but in general newly constructed or coarsely consolidated soil-road surfaces are highly irregular.

The runoff from a small catchment of road segments is considerably affected by the characteristics of surface configurations. An alternative approach to modeling surface flow must take into account the non-linearity associated with spatial variations of flows which may arise associated with spatial variations of flows which may arise from dif-

${ }^{1}$ Professor, Faculty of Agriculture, University of the Ryukyus, 1 Senbaru, Nishihara, Okinawa, 903-0216 Japan (Corresponding Author)E-mail:mshiba@agr.u-ryukyu.ac.jp

ferences of surface irregularities (Dietz et al., 1984; Shiba et al., 1986). Any flow model used to predict runoff from rainfall in these processes of water transport should include the roughness parameters related explicitly to significant characteristics of surfaces forms, especially when the model is used to analyze any changes in the surface flow due to variations in geometric properties within a drainage area (Uchida et al., 1984a;1984b).

A study is made of the possibility of representing the mode of accumulation of water on a rougher surface and development of oversurface flow by a simple flow model using computer simulation techniques. From the practical viewpoint, knowledge of drainage and alternative surfacing techniques for road maintenance would be enhanced by extending this type of study.

\section{Flow model to estimate the maximum storage capacity in depressions on a rougher road surface}

\subsection{General properties of the surface flow}

As was shown in earlier papers (Shiba et al., 1986; Shiba, 2011), the microrelief on road surface is highly irregular, and then flow over the surface is usually not steady and uniform but consists instead of intermittent slugs of flow or surges, the oversurface flow moves in trains of waves across the surface. These may be effective in increasing the erosive ability of the flow.

Irregularities on the surface also concentrate the flow in rills or in paths which, on a microscale, join together the portions of the slope in depression storage. General flow models are usually evaluated on the basis of their predictive success to the greater lengths of flow, but it is desirable that they should be applicable not only to large basins but also to small catchments and even to segments of the road sur- 
face if they are to have any explanatory power.

Although a large number of models to predict runoff from rainfall have been proposed, many of those of practical use are essentially black-box systems in which the operations to convert rainfall to runoff have no counterpart in the physical processes involved. To fit observations on all scales, a flow model must take account of all the processes involved, so that field measurement of road surface and channel characteristics may be related to model parameters.

The approach proposed here attempts to link geomorphic and hydrologic processes more closely, and demonstrates some alternative models which take account of surface flow measurements and can be applied to small catchments.

\subsection{Structure of the proposed flow model}

Figure 1 is a schematic diagram of the proposed flow model to estimate the maximum storage capacity of rainfall on the road surface. The model shows how the flow moves into irregular depressions. Here, it is assumed that two open channels are arranged at places along the cross-section at both ends of the linear segment and the surface water exceeding the maximum depression storage capacity is thus led into either of these open channels.

The computerized procedure to simulate the development of water accumulation in depressions is described as follows.

The road surface is expressed as the unit square in the $X$ - $Y$ plane and it is discretized as a $M x N$ grid of points. It is assumed that the infiltration capacity of the soil is exceeded and the continuous rainfall is considered to be of uniform intensity.
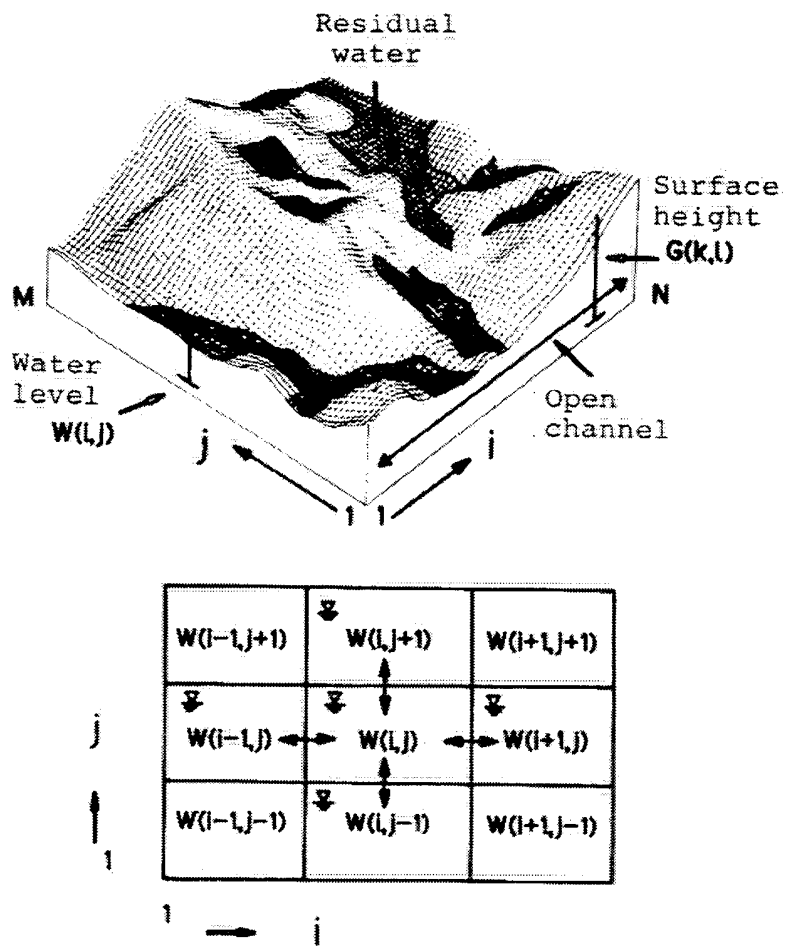

Figure 1: A schematic diagram of the proposed flow model to estimate the maximum storage capacity of rainfall on the road surface
Let us now image the amount of rainfall $R(\mathrm{~mm})$ to be uniformly given on the surface and consider the residual water to be accumulated in depressions. Denote the height distributions of surface by $G_{i, j}$ and its water level by $W_{i, j}$ which is given by $G_{i, j}$ plus $R$. Then the water level at every grid point is decided by scanning the difference between the height at the neighbors of point $(i, j)$. Here it seems reasonable that the water level at every point connected with open channels, is equal to the height of the channels, therefore the surface water exceeding the channel height is certainly led into the channel.

Hence at the initial condition of the loop on scanning the surface, the starting point $\left(i=i_{0}, j=j_{0}\right)$ is set to the first point $\left(i_{0}, j_{0}\right)$ of the channel line. All the necessary computation to estimate the water level, $W_{i, j}$ at every point $(i, j)$ in the array and its three neighbors $\left(W_{j-1, j}, W_{i, j+1}, W_{i, j-1}\right)$ in deciding singularity is performed by the following algorithm.

$W_{i+1, j}=W_{i, j}$
If $W_{i+1, j}<G_{i+1, j}$ then $W_{i+1, j}=G_{i+1, j}$

In this double loop, the new displacement array can be successively computed from the old displacement array. Now assuming that water at any point on the surface may be connected to the water level of the channel from various directions, some random point on the surface is reset to the first point, as the starting point in the double loop. Then the water level at every point in the array and its three neighbors in deciding singularity is also scanned by the following algorithm.

If

$$
\begin{aligned}
& W_{i+1, j}>W_{i, j} \text { then } W_{i+1, j}=W_{i, j} \\
& W_{i+1, j}<G_{i+1, j} \text { then } W_{i+1, j}=G_{i+1, j}
\end{aligned}
$$

Accordingly, by repeating successive calculations the residual water ration $Q(R)$ at each amount of rainfall $R(\mathrm{~mm})$ can be derived in a simple way written as:

$$
Q(R)=\frac{\sum_{i=1}^{N} \sum_{j=1}^{N}\left(W_{i, j}-G_{i, j}\right)}{R \cdot N \cdot M} \times 100
$$

where $Q(R)=$ residual water ratio $(\%)$;

$R=$ amount of the continuous rainfall;

$W_{i, j}=$ water level;

$G_{i, j}=$ surface heights;

$N$ and $M=$ the size of input matrix;

$i$ and $j=$ the index of grid points.

\section{Estimation of residual water ratio to different types of road surface}

For the function check of the proposed flow model, two types of road surface roughness $\mathrm{P}_{2}$ (Normal surfacing consolidation practice with crawler type KOMATSU bulldozer 4 ton) and $\mathrm{P}_{5}$ (control plot surface without consolidation practices) under the different condition of operational practices are tested (Shiba, 2011).

Figure 2 shows the histogram of deviation heights of two types of road surface. The diagram indicates a large difference of standard deviation between two types, which could 
be reflected in the difference of the amount of surface distortion in vertical variations along the base-plane positions. The histogram also represents a similar characteristic of both surface roughness which is a lineated flat configuration with a rougher surface, because the surface contains relatively large variation with various short wavelengths. The two distributions are reasonably symmetric and resemble normal distributions, although their tails are somewhat longer than those for theoretical normal distributions.

Figure 3 is output maps showing simulated situation of residual water after rainfall of $100 \mathrm{~mm}$ : surface roughness with $1.0 \mathrm{~cm}$ contour interval (upper), water level with 1.0 $\mathrm{cm}$ (middle) and water depth with $2.0 \mathrm{~cm}$ (lower). Estimates of water level and depth obtained by applying the flow model reflect the spatial correspondence of distributions between roughness and accumulated water in surface depressions. As a simulated model of surface flow, the proposed model would clearly passed test.

Figure 4 shows the estimates of the residual water ratio $Q(R)$ corresponding to each type of road surface (after removal of the linear trend component), which consolidated by different combinations of operations and machinery de-

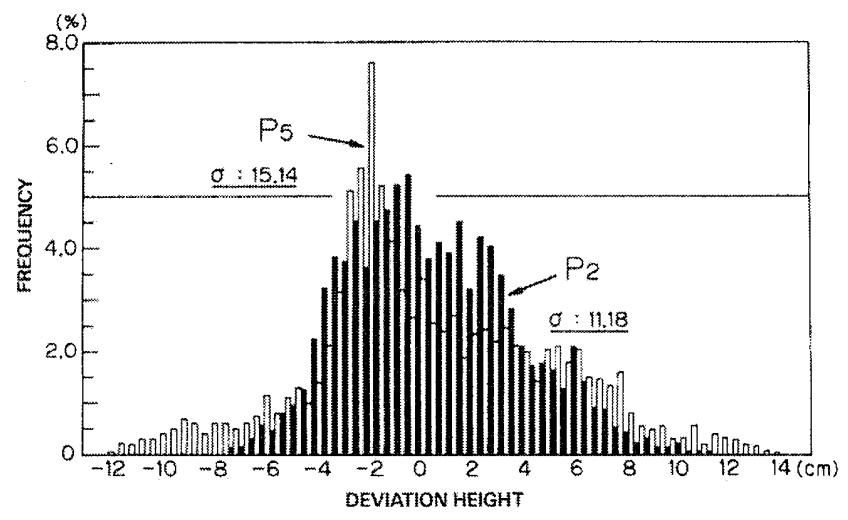

Figure 2: Histogram of deviation heights of the two types of road surface

scribed extensively in earlier paper on simulation model of road surface roughness (Shiba, 2011).

The graph indicates than an increase in rainfall is accompanied by a decrease in the residual water ratio. It appears that initially the ration is greater, although, for points of increasing rainfall, it approaches an asymptotic limit which may be defined as the 'maximum storage capacity' of the particular road surface conditions. With even these slight differences in surface roughness, each surface trend to produce the saturated accumulation of residual water in depressions preferentially. This result indicates that in potential drainability of surface $\mathrm{P}_{1}$ type has a certain advantage over other surface type.

Figure 5 shows the estimates of the residual water ratio $Q(R)$ corresponding to each type of road surface with the linear trend component included. The effect of gradient in reducing the residual water in depressions on the road surface appears more clearly in this diagram. With even slight tilting of a surface, the residual water can be considerably reduced. The extent of this tendency in $P_{1}$ and $P_{3}$ types roughly spread up to 5 to 10 percent. The effect of differ-
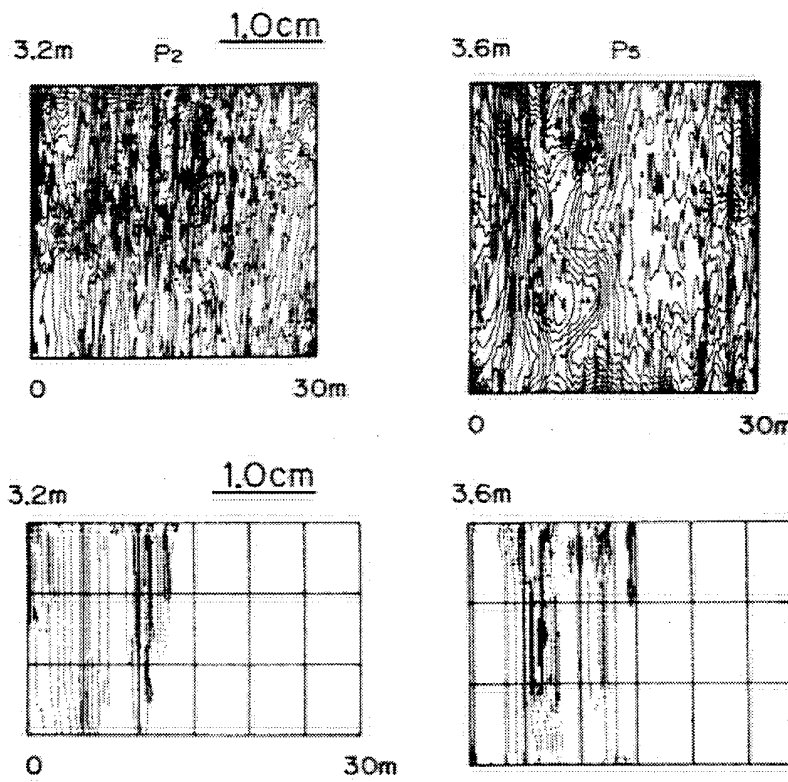

$3.6 \mathrm{~m}$
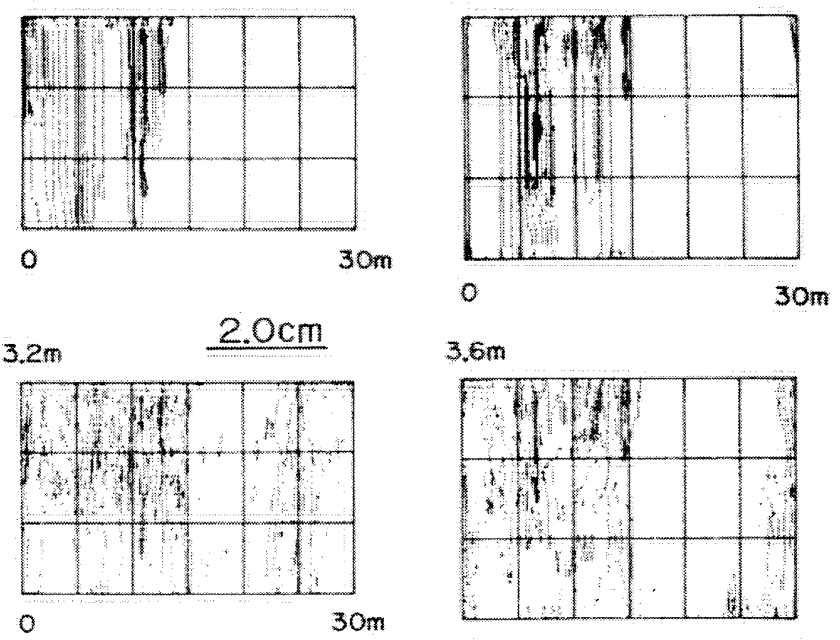

$3.6 \mathrm{~m}$

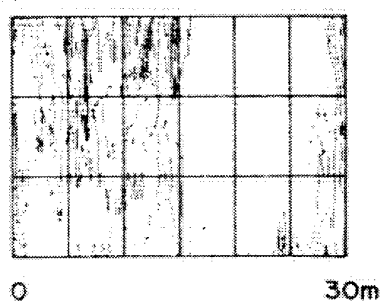

Figure 3: Test run for the function check of the proposed flow model

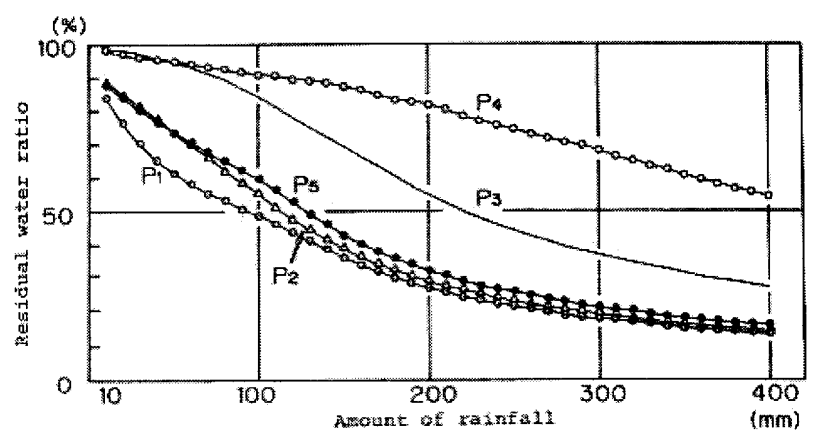

Figure 4: Estimates of the residual water ratio $Q(R)$ corresponding to each type of road surface with linear trend component removed

ences in surface roughness (including the trend component) on the residual water ratio should be noted. The effect of gradient is here only modified for surfaces with a uniform gradient or with relatively small variations of short wave-length. It may be seen that the influence of a rougher surface with long wavelengths would be to localize the high storage capacity and susceptibility to saturation by oversurface flow into the basal portion of the slope. This means that if the variation of surface roughness is relatively larger than that of the existing trend component, a sufficient reduction of the residual water in depressions by tilting the road surface may not occur because the influence of gradi- 
ent is blocked by the macrorelief of surface configurations.

Figure 6 shows the estimates of the residual water ratio $Q(R)$ obtained by applying the simulated road surface corresponding to $\mathrm{P}_{2}$ and $\mathrm{P}_{5}$ types. Estimates from the AR model reveal a remarkable difference in the residual water ratio compared with that of the measured surface, but there is no great difference between them in its decrement pattern. This is due to the fact that the first-order AR model is less capable of approximating the complicated surfaces, on a term base, than are AR models of higher orders.

These results therefore seem to require AR models of higher orders for a full quantitative description of the surface configurations (Shiba, 2011).

\section{Conclusions}

This paper presents a simple system of quantitative descriptions of drainage processes on irregular surface of forest road segments based upon computer simulation techniques, and it proposes an alternative flow model which more closely takes into account linked geomorphic and hydrologic properties. The essential particulars clarified here are as follows.

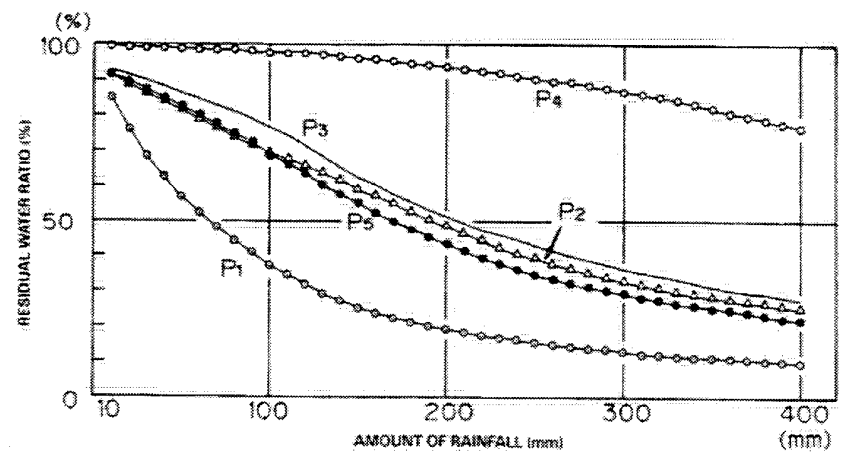

Figure 5: Estimates of the residual water ratio $Q(R)$ corresponding to each type of road surface with the linear trend component included

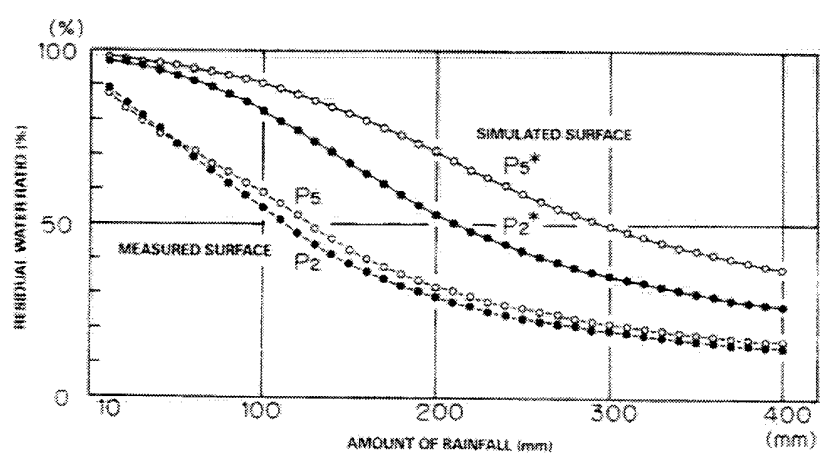

Figure 6: Estimates of the residual water ratio $Q(R)$ obtained by applying simulated road surface corresponding to $\mathrm{P}_{2}$ and $\mathrm{P}_{5}$ types
1: The simulation by flow models with various gradients and patterns of irregular depressions on a road surface, provides a basis for evaluation of the effects of tilting and smoothing on the surface consolidation.

The maximum storage capacity of a surface rapidly decreases with an increase in surface gradient, therefore, the residual water in depressions on a surface can be reduced to small amounts with even slight tilting of the trend surface.

2: The effect of differences in surface irregularities on the residual water ratio must also be noted. The effect of gradient is only modified for surfaces with an uniform gradient or with relatively small variations of short wavelength, but the effect of irregular surfaces with long wavelengths, for example, would be to localize the high storage values and susceptibility to saturation of surface flow in the slope-base portion.

This means that if the variation of roughness is relatively larger than that of a given trend surface component, a sufficient reduction of residual water in depressions by tilting a road surface may not occur. The influence of gradient is blocked by the macro- relief irregularities of the surface configuration.

\section{References}

[1] BC Code (1995): Forest Practice Code of British Columbia Act, Electronic document, http://www.for.gov.bc.ca/tasb/legsregs/fpc/fpc.htm, (accessed 2002-03-26).

[2] Coker, J., Fahey, D. and Payne, J (1993): Fine sediment production from truck traffic, Queen Charlotte Forest, Marlborough Sounds, New Zealand, J. Hydr., 31(1), pp.56-64.

[3] Dennis, D. and Heinrich, R. (1996) : FAO Model Code of Forest Harvesting Practice, Final report of the Meeting of the FAO/COFO, pp.1-85

[4] Dietz, P., Knigge, W., and Loeffler, H. (1984): Walderschkiessung, Hamburg/Berlin, pp.238-380 (in Germany).

[5] Shiba, M., Sasaki, I. and Yamamoto, T. (1986):. Computer simulation of road surface roughness and its applicability to the behavior of surface drainage. (I), Trans. 97th Ann. Meet. Jap. For. Soc.,pp. 637-640, (in Japanese).

[6] Shiba, M. (2011): Quantitative descriptions of drainage processes on irregular surface of road segments based on advanced computer simulation techniques (I) -Simulation of road surface roughness using AR model-, pp.1-9, submitted paper to JRCS.

[7] Silversides, R. and Sundberg U. (1989): Operational Efficiency in Forestry: Volume 2: Practice, The Netherlands, pp.2105-131.

[8] Uchida, H., Maruyama, T., and Kobayashi, S. (1984a): Basic Study on Two Dimensional Roughess, Trans. JSIDER $112,1-8$, (in Japanese).

[9] Uchida, H., Maruyama, T., and Kobayashi, S. (1984b) Basic Study on Three Dimensional Roughness, Trans. JSIDER 114, 1-6, (in Japanese).

[10] Visser, R. and Smith, M.(1993) New Zealand Forest Code of Practice (Second Edition), LIRO report, Rotorua, pp.1-103

Discussion open until Dcember 31, 2012 